\title{
X-ray spectroscopic studies of the electronic structure of chromium-based $p$-type transparent conducting oxides
}

\author{
E. Norton,${ }^{1,2, *}$ L. Farrell, ${ }^{1,2}$ S. D. Callaghan,${ }^{1}$ C. McGuinness, ${ }^{1}$ I. V. Shvets, ${ }^{1,2}$ and K. Fleischer ${ }^{1,2}$ \\ ${ }^{1}$ School of Physics, Trinity College, University of Dublin, Dublin 2, Ireland \\ ${ }^{2}$ Centre for Research on Adaptive Nanostructures and Nanodevices (CRANN)
}

(Received 8 January 2016; revised manuscript received 17 February 2016; published 7 March 2016)

\begin{abstract}
The valence band structure of $p$-type transparent oxides-crystalline $\mathrm{Mg}_{x} \mathrm{Cr}_{2-x} \mathrm{O}_{3}$ and nanocrystalline $\mathrm{Cu}_{x} \mathrm{CrO}_{y}$-is analyzed as a function of incoming photon energy. The valence band of both $p$-type transparent conducting oxides shows striking similarities to measurements on crystalline $\mathrm{CuCrO}_{2}: \mathrm{Mg}$ with all films showing that chromium states compose the top of the valence band, suggesting that the valence-band structure is dominated by the presence of the $\mathrm{Cr}^{-} \mathrm{O}_{6}$ octahedra. A comparison of the valence band between the best performing $p$-type, crystalline $\mathrm{CuCrO}_{2}: \mathrm{Mg}$, with crystalline $\mathrm{Mg}_{x} \mathrm{Cr}_{2-x} \mathrm{O}_{3}$ and nanocrystalline $\mathrm{Cu}_{x} \mathrm{CrO}_{y}$ shows that the chromium $3 d$ states are fixed irrespective of changes in long-range crystallographic order. This indicates little spatial overlap between adjacent $\mathrm{Cr} 3 d$ states. This further confirms the conduction mechanism via hopping for chromium based $p$-type TCOs as the $\mathrm{Cr} 3 d$ states are localized within the $\mathrm{Cr}-\mathrm{O}_{6}$ octahedra.
\end{abstract}

DOI: 10.1103/PhysRevB.93.115302

\section{INTRODUCTION}

Optically transparent conductive materials (TCMs) such as conductive polymers, 2D materials like graphene, nanowires, and metal oxides offer the promise of fully transparent electronic devices [1,2]. Transparent conducting metal oxides (TCOs) are the most widely used TCMs by industry due to the excellent properties of $n$-type TCOs-indium tin oxide (ITO), fluorine-doped oxide (FTO), and aluminium-doped zinc oxide (AZO) [3]. However, fully transparent devices have been hindered by $p$-type TCOs having poor performance in terms of carrier mobility and transparency [4-7]. Moreover, very few materials show promise of stable bipolar doping [6,8], which if successful would open up the possibility of optically transparent CMOS technology [9].

In $p$-type TCOs, the main challenge has been to overcome that the valence-band maximum is composed largely of states with oxygen $2 p$ character, which will hinder high mobility holes, converse to the $s$-like state conduction band minimum of metal oxides. The selection of an open $d$-shell transition metal has been crucial to obtain good performing $p$-type conductivity in TCOs [10]. Since the initial report by Kawazoe et al. [11], delafossites have attracted much attention as $p$-type TCO materials. The delafossite structure, $A B \mathrm{O}_{2}$, has an $A$ cation site linearly coordinated to the oxygen octahedra within which the $\mathrm{B}$ site cation resides. In particular, cuprous delafossites exhibit exceptional electrical, magnetic, and optical properties [12].

However, $p$-type films suffer from low mobilities and can typically only be estimated by the Seebeck effect. There are some exceptions such as $\mathrm{CuCrO}_{2}: \mathrm{Mg}$, which was measured by the AC Hall effect [13]. This limits their use in applications such as transparent transistors where mobility is paramount. Beyond the low mobility, $p$-type TCOs have relevance in photovoltaics as selective contacts for the extraction of holes to improve the overall efficiency and performance of solar cells [14-17]. Furthermore, a recent paper by Chen et al. shows $p$-type TCOs to be useful as a selective contact as well as a

\footnotetext{
*nortonem@tcd.ie
}

protective overlayer with $n$-type silicon for stable, efficient solar water splitting [18].

An appropriate figure of merit (FOM) to characterize the performance of a TCO is a ratio of electrical conductivity weighted against the optical absorption coefficient [19]. $\mathrm{CuCrO}_{2}: \mathrm{Mg}$ exhibits the highest FOM to date [20]. The success of the cuprous delafossites in exhibiting exceptional electrical properties was attributed to copper $3 d$ states reducing the oxygen $2 p$ character at the top of the valence band through hybridization. Initially, the choice of chromium as the optimum $B$ site cation was attributed to closed lattice matching of the two cation sites, which affected the optical properties fortuitously. Interestingly, a recent paper by Farrell et al. reports a copper deficient nanocrystalline $\mathrm{CuCrO}_{2}$ [22] material with an FOM of $350 \mu \mathrm{S}$ and conductivity as high as $12 \mathrm{~S} \mathrm{~cm}^{-1}$. The $\mathrm{Cu}_{x} \mathrm{CrO}_{y}$ films have an FOM an order of magnitude greater than $\mathrm{CuCrO}_{2}: \mathrm{Mg}$ deposited previously by spray pyrolysis and also only an order of magnitude below the best FOM reported for $\mathrm{CuCrO}_{2}: \mathrm{Mg}$ [23].

Theoretical studies of the electronic structure of $p$-type $\mathrm{CuCrO}_{2}$ led to conflicting reports on the nature of the top of the valence band: whether it is predominately composed of $\mathrm{Cu}$ or $\mathrm{Cr} 3 d$ states. The first reports from theoretical and experimental studies suggested that $\mathrm{Cu}$ states lay at the top of the valence band [24-26]. Further DFT $+U$ studies by Scanlon and Watson, and experimental confirmation by Yokobori et al. using resonant valence-band photoemission spectroscopy (RVBPES) showed evidence that in fact chromium states predominately compose the top of the valence band $[27,28]$.

Furthermore, a large range of chromium based $p$ type TCOs have been reported in the last 5 years: two corundum lattice structures- $-\mathrm{Mg}_{x} \mathrm{Cr}_{2-x} \mathrm{O}_{3}$ [21,29] and $\mathrm{Ni}_{x} \mathrm{Cr}_{2-x} \mathrm{O}_{3}$ [30] —have been shown to be $p$-type TCOs. Several other reports of high figure of merit (FOM) $p$-type TCOs also contain chromium in an octahedral coordination. Besides the prototypical $\mathrm{Mg}_{x} \mathrm{Cr}_{2-x} \mathrm{O}_{3}$ and $\mathrm{Ni}_{x} \mathrm{Cr}_{2-x} \mathrm{O}_{3}$, these include $\mathrm{LaCrO}_{3}: \mathrm{Sr}$ [31] and spinel structures such as $\mathrm{Cr}_{2} \mathrm{MnO}_{4}: \mathrm{Li}[32,33]$.

In this study, RVBPES has been carried out on films of $\mathrm{Mg}_{x} \mathrm{Cr}_{2-x} \mathrm{O}_{3}$ and $\mathrm{Cu}_{x} \mathrm{CrO}_{y}$ with varying $\mathrm{Cu} / \mathrm{Cr}$ ratio, 


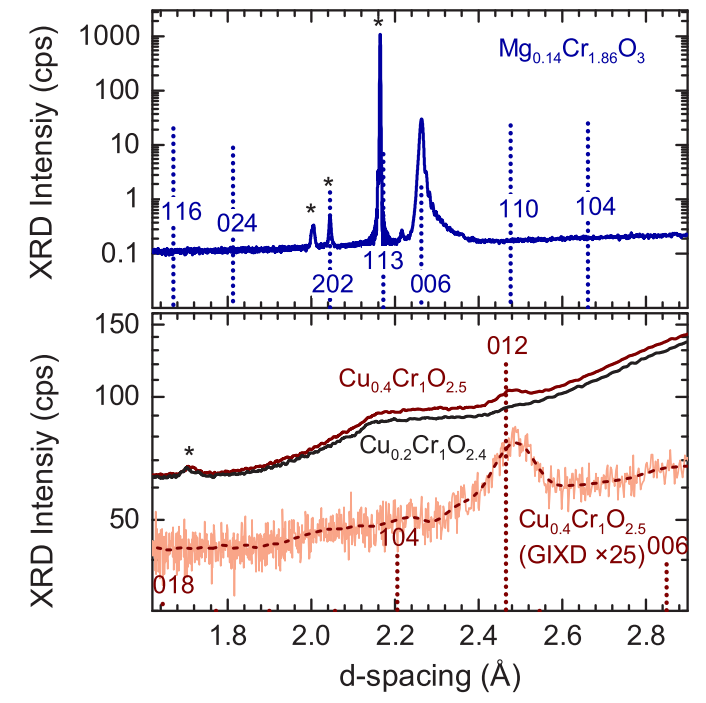

FIG. 1. X-ray diffraction of the analyzed samples. The top panel shows the epitaxially grown $\mathrm{Mg}_{x} \mathrm{Cr}_{2-x} \mathrm{O}_{3}$. Only the 006 reflex of the eskolaite phase is observed. The lower panel shows the data for the nanocrystalline $\mathrm{Cu}_{x} \mathrm{CrO}_{y}$. To illustrate the polycrystalline nature, a symmetric $\theta / 2 \theta$ measurement is compared to a grazing incidence measurement. Only the strongest $\mathrm{CuCrO}_{2}$ reflex can be observed for the sample with higher copper content. Structures originating from the substrate and/or sample holders have been marked (*).

providing qualitative experimental evidence about the composition of the valence band. Experimentally, we can probe this by using resonant photoemission spectroscopy (RPES). RPES is a technique that is often described as a two-step process: the creation of a core hole, and secondly the decay of that core hole. The decay of the core hole causes the emission of an Auger electron from the valence band. This valence electron has the same final state as an electron emitted by direct photoemission. However, these studies ideally have films that are sufficiently conductive to avoid shifts in binding energies due to sample charging, hence only highly conductive $p$-TCOs can be easily analyzed.

While several studies have been performed on crystalline $\mathrm{CuCrO}_{2}: \mathrm{Mg}$ a comparison with $\mathrm{Mg}_{x} \mathrm{Cr}_{2-x} \mathrm{O}_{3}$ and $\mathrm{Cu}_{x} \mathrm{CrO}_{y}$ will provide a general insight into the electronic structure and low mobility of chromium based $p$-type TCOs. Few studies of this nature have been carried out on conducting oxides. The $\mathrm{Cu}_{x} \mathrm{CrO}_{y}$ films, an oxide synthesized at low temperatures, has been shown to be copper deficient with poor crystalline quality, yet possesses a high FOM. The defective material allows us, by altering the $\mathrm{Cu} / \mathrm{Cr}$ ratio, to switch between two regions: a highly copper doped amorphous $\mathrm{Cr}_{2} \mathrm{O}_{3}: \mathrm{Cu}$ structure and a copper deficient $\mathrm{CuCrO}_{2}$. While less conductive than the crystalline $\mathrm{CuCrO}_{2}: \mathrm{Mg}$, it has one of the highest FOMs, $350 \mu \mathrm{S}$, of any solution processed $p$-type TCOs [34].

The difference in the crystallographic properties is illustrated in the $\mathrm{x}$-ray diffraction (XRD) patterns of the samples shown in Fig. 1. The $\mathrm{Mg}_{x} \mathrm{Cr}_{2-x} \mathrm{O}_{3}$ sample is epitaxially grown on $c$ plane $\mathrm{Al}_{2} \mathrm{O}_{3}$ showing only the 006 reflex of the eskolaite phase. In contrast, the $\mathrm{Cu}_{x} \mathrm{CrO}_{y}$ samples are nanocrystalline samples with a coherent domain size below $8 \mathrm{~nm}$ and no preferential growth direction. The phase identification in this case is difficult by XRD alone as the film signal is weak compared to the substrate and sample holder background. A more comprehensive study on the crystallographic properties by XRD and Raman spectroscopy has been published elsewhere [34]. Despite the poor crystallinity, the films are closed, smooth layers and can be readily incorporated in devices.

\section{EXPERIMENTAL METHODS}

Thin film samples of chromium oxide based $p$-type TCOs have been prepared by either molecular beam epitaxy $\left(\mathrm{Cr}_{2} \mathrm{O}_{3}\right.$ based, crystalline films) or spray pyrolysis (copper deficient $\left.\mathrm{CuCrO}_{2}\right)$. The $\mathrm{Mg}_{x} \mathrm{Cr}_{2-x} \mathrm{O}_{3}$ films were grown on $\mathrm{Al}_{2} \mathrm{O}_{3}(0001)$ substrates using a DCA M600 molecular beam epitaxy system (MBE) with base pressure of $1.3 \times 10^{-7} \mathrm{~Pa}$. E-beam evaporation was done from $\mathrm{Cr}_{2} \mathrm{O}_{3}$ pellets (99.9\% Kurt $\mathrm{J}$. Lesker) at an oxygen partial pressure $6 \times 10^{-3} \mathrm{~Pa}$ with the substrate temperature maintained at $873 \mathrm{~K}$. The doping was achieved by controlling the supplied power to a second e-beam evaporator loaded with $\mathrm{MgO}$ (99.95\% Kurt J. Lesker) [21]. After growth, the $\mathrm{Mg}_{x} \mathrm{Cr}_{2-x} \mathrm{O}_{3}$ film was postannealed in oxygen for $2 \mathrm{~h}$ at a temperature of $973 \mathrm{~K}$, showing an FOM of $3.5 \mu \mathrm{S}\left(\sigma \approx 0.04 \mathrm{~S} \mathrm{~cm}^{-1}\right)$, a factor of three improvement on the as-grown film $[21,35]$. The $\mathrm{Mg}_{x} \mathrm{Cr}_{2-x} \mathrm{O}_{3}$ film showed a composition of $\mathrm{Mg}_{0.12} \mathrm{Cr}_{1.86} \mathrm{O}_{3}$ when characterized by XPS, using a laboratory-based Omicron multiprobe system with monochromatic $\mathrm{Al} K_{\alpha}$ x rays $(h v=1486.7 \mathrm{eV})$ and an EA125 analyzer.

Nanocrystalline, highly conductive, copper-deficient copper chromium oxide was grown by spray pyrolysis using a simple air blast nozzle. Copper(II) acetylacetonate and chromium(III) acetylacetonate precursors in a methanol solution were sprayed at $623 \mathrm{~K}$ on glass microscope slides [34]. The incorporated copper content of the $\mathrm{Cu}_{x} \mathrm{CrO}_{y}$ films was controlled by altering the concentration of copper acetylacetonate in the initial precursor solution. The samples examined in this study are a high FOM $\left(150 \mu \mathrm{S}, \sigma \approx 5 \mathrm{~S} \mathrm{~cm}^{-1}\right)$ nanocrystalline film with a copper deficient composition, $\mathrm{Cu}_{0.40} \mathrm{Cr}_{1} \mathrm{O}_{2.5}$, and a lower FOM ( $\left.4 \mu \mathrm{S}, \sigma \approx 0.15 \mathrm{~S} \mathrm{~cm}^{-1}\right)$ film, which corresponds to a lower copper content, $\mathrm{Cu}_{0.2} \mathrm{Cr}_{1} \mathrm{O}_{2.4}$. X-ray absorption spectroscopy (XAS), additional core level x-ray photoemission spectroscopy (XPS), and resonant valenceband photoemission spectroscopy (RPES) were carried out at the D1011 beamline at MAX II, MAX-lab, Sweden. All photoemission spectra were normalized by the ring current and the beam line transfer function measured by a reference total electron yield on a clean gold mesh. The valence-band spectra at photon energies of 40-100 eV were taken with a fixed exit slit and a photon flux of between $10^{11}-10^{12} \mathrm{ph} / \mathrm{s}$ incident on the sample. The binding energies in valence-band spectra were aligned to the Fermi edge taken of tantalum foil in contact with the sample. All XAS absorption spectra were taken in total electron yield (TEY) mode by measuring the drain current as a function of incident photon energy. XRD patterns have been measured with a Bruker D8 Discover using a monochromated $\mathrm{Cu}-K_{\alpha}$ source (epitaxial film and grazing incidence measurement) as well as a Bruker D8 Advance using a Mo- $K_{\alpha}$ source (Powder XRD for nanocrystalline films). $\mathrm{Cr}_{2} \mathrm{O}_{3}$ atomic multiplet calculations were carried out with the CTM4XAS software [36]. 


\section{RESULTS}

\section{A. Surface preparation}

The deposition methods required to prepare $\mathrm{Mg}_{x} \mathrm{Cr}_{2-x} \mathrm{O}_{3}$ and $\mathrm{CuCrO}_{2}$ films limit studies to ex situ characterization. As-grown films are contaminated by surface carbon obscuring the measurement of the valence-band spectra. Hence all films were Ar sputtered with a beam energy of $1 \mathrm{keV}$ at $4 \times 10^{-4} \mathrm{~Pa}$ at grazing incidence to remove carbon and residuals of unreacted precursors from the surface prior to measurements. This was repeated in 4-minute steps at a time until a surface sensitive XPS scan of the $\mathrm{C} 1 s$ core level taken at an incident photon energy of $380 \mathrm{eV}$ would reveal minimal or no surface carbon present.

However, particularly in the case of $\mathrm{CuCrO}_{2}$, valence band changes have been reported in crystalline thin films due to surface preparation [12]. As our $\mathrm{Cu}_{x} \mathrm{CrO}_{y}$ thin films on glass are polycrystalline in nature, they will not have a coherent surface termination unlike in situ cleaved single crystals. Therefore introducing further disorder is unlikely to produce spectral differences in the valence band unlike in the case of Shin et al. studying crystalline $\mathrm{CuCrO}_{2}$. Nevertheless, the effects of argon sputtering and annealing on the core levels and absorption spectra is discussed to quantify any differences.

X-ray photoelectron spectroscopy (XPS), Fig. 2, shows little change in the core level of the copper $2 p_{3 / 2}$ peak in the $\mathrm{Cu}_{x} \mathrm{CrO}_{y}$ thin films due to sputtering and annealing. The only observable difference is an increase in the asymmetry of the copper peak after oxygen annealing with greater weight at the low binding energy side.

This observation correlates with significant changes in the copper $L_{2,3}$ edge, (Fig. 2) with oxygen annealing. The prepeak at $h v \approx 926 \mathrm{eV}$, which has been reported as an XAS final state of $2 p 3 d^{10}$, which corresponds to an initial $3 d^{9}$ state, i.e., $\mathrm{Cu}^{2+}$ impurities, is observed in the as-grown films and re-emerges with oxygen annealing [37]. The same prepeak, but significantly reduced in argon sputtered samples, is observed for $\mathrm{Mg}$ doped $\mathrm{CuCrO}_{2}$ and stoichiometric $\mathrm{CuCrO}_{2}$ [26,28]. In our $\mathrm{Cu}_{x} \mathrm{CrO}_{y}$ films, it decreases with, but is not eliminated by argon sputtering and re-appears with oxygen annealing. The residual peak after sputtering is representative of the bulk film which has been characterised extensively in previous studies and seems consistent with $\mathrm{CuCrO}_{2}: \mathrm{Mg}[21,34]$. We suggest that due to the defective nature of our films the surface sites are preferentially oxidised, changing the copper oxidisation state locally on the surface but not in the bulk.

\section{B. X-ray absorption spectroscopy}

X-ray absorption spectroscopy (XAS) was performed on all films, after surface preparation. Soft $x$ rays in the photon energy range of 40-1500 $\mathrm{eV}$ allowed us to probe the $\mathrm{Cr} L$-edge absorption in $\mathrm{Cu}_{x} \mathrm{CrO}_{y}$ and $\mathrm{Mg}_{x} \mathrm{Cr}_{2-x} \mathrm{O}_{3}$ films as well as the $\mathrm{Cu} L$ edge in $\mathrm{Cu}_{x} \mathrm{CrO}_{y}$ films (see Fig. 3).

All absorption spectra have been normalised to the maximum intensity after background subtraction to compare features. A reference spectra of an epitaxial $\mathrm{Cr}_{2} \mathrm{O}_{3}$ film was taken; comparison with our $\mathrm{Mg}_{x} \mathrm{Cr}_{2-x} \mathrm{O}_{3}$ and $\mathrm{Cu}_{x} \mathrm{CrO}_{y}$ films shows the chromium oxidation state unchanged. The $\mathrm{Cr} L$ edge for both $\mathrm{Mg}_{x} \mathrm{Cr}_{2-x} \mathrm{O}_{3}$ and $\mathrm{Cr}_{2} \mathrm{O}_{3}$ show an overall sharper structure than in the $\mathrm{Cu}_{x} \mathrm{CrO}_{y}$ films. The epitaxial nature of the $\mathrm{Mg}_{x} \mathrm{Cr}_{2-x} \mathrm{O}_{3}$ creates a more ordered local coordination of the chromium atoms than the nanocrystalline $\mathrm{Cu}_{x} \mathrm{CrO}_{y}$ with random crystal axis orientation. Spectral features remain consistent across the films though.

A reduction in intensity of the $\mathrm{Cu}$ L edge is seen for different films, which correlates with a reduction in $\mathrm{Cu}$ content from $\mathrm{Cu}_{0.2} \mathrm{Cr}_{1} \mathrm{O}_{2.4}$ compared to the higher FOM, $\mathrm{Cu}_{0.4} \mathrm{Cr}_{1} \mathrm{O}_{2.5}$ film. Unlike the chromium absorption spectra, the assignment of a clear oxidation state and electronic structure with a cuprite phase, $\mathrm{Cu}_{2} \mathrm{O}\left(\mathrm{Cu}^{+1}\right)$, is difficult. The copper $\mathrm{x}$-ray absorption spectra has been notoriously difficult to analyze. Nominally, this oxidation state will have fully occupied copper $3 d$ states in the valence band and an empty $\mathrm{Cu} 4 s$ conduction band. The
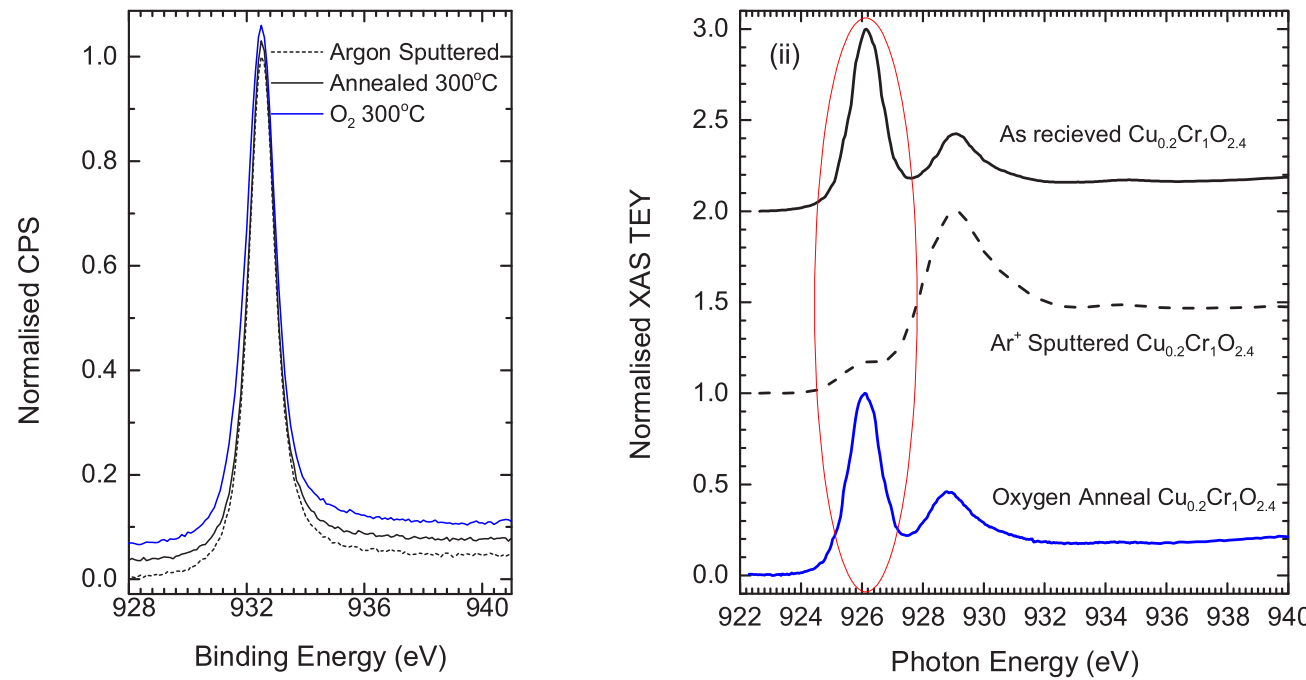

FIG. 2. (i) X-ray photoelectron spectroscopy of $\mathrm{CuCrO}_{2}$ films highlighting the core level changes when the sample is argon sputtered and annealed in oxygen. A slight increase in the peak asymmetry is noted when the film is annealed in oxygen. (ii) In contrast, spectra of the $\mathrm{Cu}$ $L_{2,3}$ edge as a function of surface preparation. A large pre-peak, circled, is observed at $h v=926 \mathrm{eV}$ in as received $\mathrm{Cu}_{x} \mathrm{CrO}_{y}$ films. $\mathrm{Argon}$ sputtering reduces, but does not eliminate this peak entirely. Oxygen annealing after argon sputtering reproduces the same prepeak. 

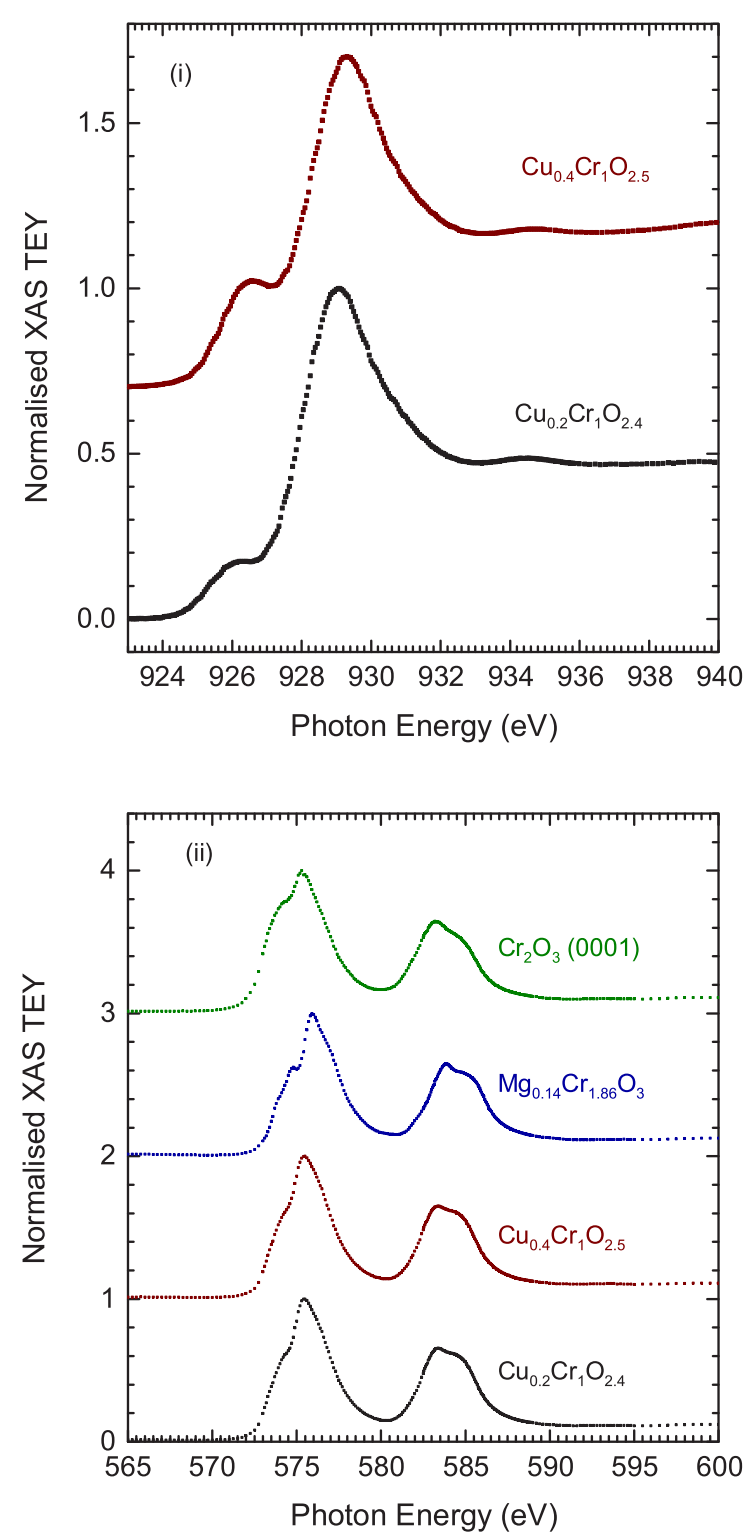

FIG. 3. (i) X-ray absorption spectra of the $\mathrm{Cu} L_{2,3}$ of $\mathrm{Cu}_{0.2} \mathrm{Cr}_{1} \mathrm{O}_{2.4}$ and $\mathrm{Cu}_{0.4} \mathrm{Cr}_{1} \mathrm{O}_{2.5}$ and (ii) $\mathrm{Cr} L_{2,3}$ edge of $\mathrm{Cu}_{0.2} \mathrm{Cr}_{1} \mathrm{O}_{2.4}, \mathrm{Cu}_{0.4} \mathrm{Cr}_{1} \mathrm{O}_{2.5}$, and $\mathrm{Mg}_{x} \mathrm{Cr}_{2-x} \mathrm{O}_{3}$.

band-structure calculations show $\mathrm{Cu}_{2} \mathrm{O}$ to have substantial $3 d$ character in the conduction band and conversely $4 s$ character in the valence band [38]. However, experimentally the $\mathrm{Cu} L$ edge is difficult to analyze as $\mathrm{x}$-ray spectroscopic investigations are nearly always overly sensitive to a mixed ground state as $2 p$ edge core level spectroscopy is dominated by atomic multiplet effects as noted for instance in resonant inelastic $\mathrm{X}$-ray spectra (RIXS) of $\mathrm{Cu}_{2} \mathrm{O}$ being dominated by $3 d \rightarrow 3 d^{*}$ transitions [39].

In Fig. 3, the structure in the $\mathrm{Cu}$ L-edge around $926 \mathrm{eV}$ is found to be more intense in the better conducting $\mathrm{Cu}_{0.4} \mathrm{Cr}_{1} \mathrm{O}_{2.5}$ sample even after sputtering. A similar structure was found to appear in $\mathrm{Mg}$ doped $\mathrm{CuCrO}_{2}$, increasing in intensity with magnesium concentration by Yokobori et al. [28]. The appearance of the same structure in copper deficient material suggests that the origin of the peak is a copper atom in a different electronic configuration in some defect complex involving the copper site and, e.g., an adjacent oxygen interstitial $\left(\mathrm{O}_{i}\right)$. In $\mathrm{CuCrO}_{2}$, these defect complexes might be generated by magnesium substitution, while they natively occur in our defective $\mathrm{Cu}_{x} \mathrm{CrO}_{y}$.

DFT calculations of single point defects in $\mathrm{CuCrO}_{2}$ have shown that $\mathrm{V}_{\mathrm{Cu}}$ are the most readily formed intrinsic defects, while $\mathrm{Mg}_{\mathrm{Cu}}$ is the dominant defect in doped materials [27]. However, no evaluation of complex defects such as $\mathrm{Mg}_{\mathrm{Cu}}-\mathrm{V}_{\mathrm{Cu}}$ or $\mathrm{V}_{\mathrm{Cu}}-\mathrm{O}_{i}$ has been performed yet. In the related $\mathrm{Cu}_{2} \mathrm{O}$, it has already been shown that the formation of defect complexes readily occurs, explaining, e.g., why $\mathrm{Sr}^{+2}$ doping in $\mathrm{Cu}_{2} \mathrm{O}$ leads to $p$-type conductivity as the $p$-type $\mathrm{Sr}_{\mathrm{Cu}}-\mathrm{V}_{\mathrm{Cu}}$ defect complex is more likely to form than the $n$-type $\mathrm{Sr}_{\mathrm{Cu}}$ isolated point defect [40]. In a similar fashion, other native defect complexes in $\mathrm{CuCrO}_{2}$ could be contributing factors. The identification of their detailed nature would require $a b$ initio analysis of many possible combinations including $\mathrm{Cr}_{\mathrm{Cu}}-\mathrm{V}_{\mathrm{Cu}}, \mathrm{O}_{i}-\mathrm{V}_{\mathrm{Cu}}$ and others. The observed structure for surfaces exposed to air or oxygen annealing would indicate an increase in the number of some defect like complexes. The fact that this increase is not observed by vacuum annealing alone and the $\mathrm{Cr} L$ edge is not affected leads to the identification of a $\mathrm{Cu}-\mathrm{O}_{i}$ defect complex as a likely candidate not only for the changed surface termination but also as a contributing factor in the bulk doping of the material itself.

\section{Resonant photoelectron spectroscopy}

Figure 4 outlines the valence-band spectra of the $\mathrm{Mg}_{x} \mathrm{Cr}_{2-x} \mathrm{O}_{3}$ film over a range of photon energies. Two possible processes leading to the final state $3 d^{n-1}$ are possible. The direct process of emitting a valence electron is possible for all incident photon energies greater than the valence electron binding energy,

PES: $3 d^{n}+h v \rightarrow 3 d^{n-1}+\mathrm{e}^{-1}$.

However, if we have a tunable photon energy, we can generate a second process, outlined below for a $3 p \rightarrow 3 d$ transition:

RPES: $3 d^{n}+h v \rightarrow 3 p^{5 \curvearrowright} 3 d^{n+1} \rightarrow 3 p^{6} 3 d^{n-1}+\mathrm{e}^{-1}$.

While directly photoemitted electrons (PES) provide nonselective sampling of the atomic electronic levels, resonant photoemission spectroscopy (RPES) can be used to probe them selectively. By taking the same valence-band spectra collected at different photon energies, any increase in the intensity of a specific feature would indicate an increase, in our case, of $3 d^{n-1}$ electrons emitted by photoemission through the intermediate resonant process.

Figure 4(ii) plots the valence-band photoemission intensity at a given binding energy as a function of the photon energy over the $3 p-3 d$ threshold and upwards. At the top of the valence band, two resonance features of interest are seen: a weak spectral response at 44-46 eV (red box) at a binding energy of $2.7 \mathrm{eV}$ and a stronger resonant behavior at 48-50 eV (black box) at a slightly higher binding energy of $3 \mathrm{eV}$. The expected $3 p-3 d$ spectrum can be modeled using CTM4XAS software so we can see if the origin of this double peak feature can be pinpointed [36]. For the atomic multiplet calculation the value of the crystal-field parameter, $10 D_{q}$, was chosen to be $2.03 \mathrm{eV}$; a value derived from the optical $2 \mathrm{~T}_{g}-2 \mathrm{~A}_{g}$ transition observed 

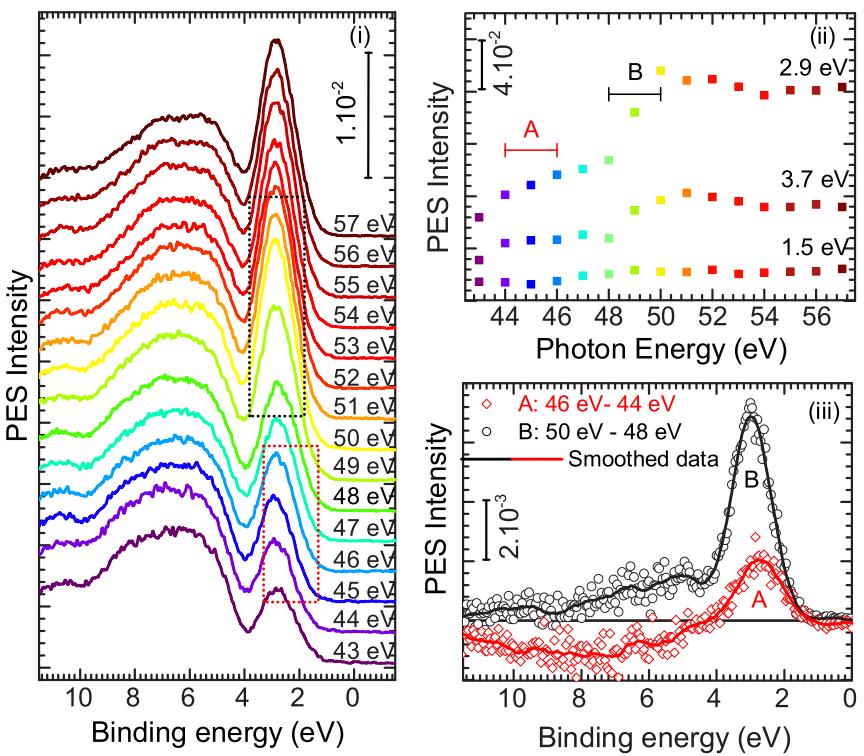

FIG. 4. (i) Valence-band spectra of the $\mathrm{Mg}_{0.12} \mathrm{Cr}_{1.86} \mathrm{O}_{3}$ film over the photon energies 43 to $57 \mathrm{eV}$ with an FOM of $3.5 \mu \mathrm{S}$. The black box in (i) indicates the region where resonant behavior over $48-50 \mathrm{eV}$ is observed. The red box indicates the lower resonance over $44-46 \mathrm{eV}$. (i) PES intensity for selected binding energies as a function of incident photon energy. The strong enhancement around $49 \mathrm{eV}$ is caused by a resonance to $\mathrm{Cr} 3 p-3 d$ transitions. A weaker enhancement is seen around $45 \mathrm{eV}$. (iii) Differences between the spectra at 50 and $48 \mathrm{eV}$ (०) reveal the strong $\mathrm{Cr}$ resonance around $3 \mathrm{eV}$ and an increase of PES intensity of 0.007 . The spectral difference between 46 and $44 \mathrm{eV}(\diamond)$ shows the onset of that resonance with reduced PES intensity (0.002). The negative differential signal in the $4-8 \mathrm{eV}$ range is caused from going off-resonance to the oxygen $p$ states.

in the pure epitaxially grown $\mathrm{Cr}_{2} \mathrm{O}_{3}$ films. This crystal-field splitting is broadly consistent with that observed from pure single crystals of $\mathrm{Cr}_{2} \mathrm{O}_{3}$ where it was found to be $2.08 \mathrm{eV}$ [41]. Although a trigonal distortion away from $\mathrm{O}_{h}$ to $\mathrm{C}_{3}$ symmetry is present in the corundrum lattice, the effect of such a distortion on the optical spectra is very small and various cluster and multiplet calculations effectively ignore this distortion as a result $[42,43]$.

The Cr $2 p$ and $3 p$ XPS, $L_{3,2}$ and $M_{3,2}$ XAS are all best modelled taking into account charge transfer multiplets. A model for such charge transfer parameters has been previously put forward by Matsubara et al. [44] who successfully modeled $\mathrm{Cr}_{2} \mathrm{O}_{3}$ XAS, $2 p$ XPS, and $2 p-3 d$ resonant inelastic x-ray scattering (RIXS) data. These same charge transfer energies are used in the present modeling. The $L_{3,2}$ XAS and $2 p$ XPS are modeled with core hole lifetimes for the $L_{3} 2 p_{3 / 2}$ hole of $0.32 \mathrm{eV}$ and the $L_{2} 2 p_{1 / 2}$ hole of $0.76 \mathrm{eV}$ as recommended by Campbell and Papp [45], with an instrumental broadening of $0.1 \mathrm{eV}$ and $0.3 \mathrm{eV}$, respectively. Our model reproduces the expected spectral features seen in the Cr $2 p$ and $3 p$ XPS, and $L_{3,2}$ XAS.

Figure 5 shows a plot of the $M_{3,2}$ absorption edge. This edge and $3 p$ XPS are modeled with core hole lifetimes of $1.2 \mathrm{eV}$ for the $M_{3,2 / 3 p}$ holes and similar instrumental broadening. In Fig. 5, two features A and B are present in the $3 p$ XAS spectra. Feature A, the lower excitation energies of $40-45 \mathrm{eV}$, is due to

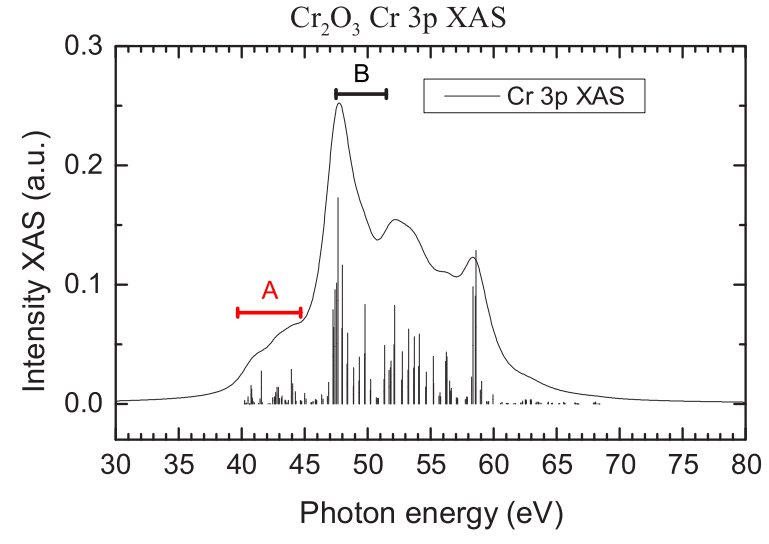

FIG. 5. Cr $M_{3,2}$ XAS spectrum with the distribution of intensities as obtained in a charge transfer atomic multiplet calculation. Feature A: $40-45 \mathrm{eV}$ is due to a dominant transition to a low-spin XAS final state of $2 p^{5} 3 d^{4}\left(\mathrm{t}_{2 g}^{4}\right)$. The more intense feature $\mathrm{B}$ at higher excitation energies (49-55 eV) is the much more strongly allowed high-spin XAS final state of $2 p^{5} 3 d^{4}\left(\mathrm{t}_{2 g}^{3} \mathrm{e}_{g}^{1}\right)$.

a dominant transition to a low spin XAS final state of $2 p^{5} 3 d^{4}$ $\left(\mathrm{t}_{2 g}^{4}\right)$. The more intense feature, B, at higher excitation energies $(49-55 \mathrm{eV})$ is the much more strongly allowed high spin XAS final state of $2 p^{5} 3 d^{4}\left(\mathrm{t}_{2 g}^{3} \mathrm{e}_{g}^{1}\right)$ as previously remarked by both Li et al. and Bertel et al. [46,47]. In the case of the $M_{3,2}$ edge, the atomic multiplet nature of the transitions dominate the intensity profile interlinked with the density and intensity of the allowed transitions to the given XAS final states. Although these are the XAS final states, they are the intermediate state in the $3 p-3 d$ VB RXPS spectrum and thus govern at what excitation energy the observed VB RXPS occurs at and also how intense that feature will be. The origin of the features $\mathrm{A}$ and B in Fig. 4 in the $\mathrm{Mg}_{x} \mathrm{Cr}_{2-x} \mathrm{O}_{3}$ film across the $3 p-3 d$ resonance can be linked to a difference in energy between the high- and low-spin states $\left(\mathrm{t}_{2 g}^{4}\right)$ and $\left(\mathrm{t}_{2 g}^{3} \mathrm{e}_{g}^{1}\right)$, which are the intermediate states for RPES.

Figure 6 shows similar measurements for the investigated $\mathrm{Cu}_{x} \mathrm{CrO}_{y}$ films over the same $\mathrm{Cr} 3 p-3 d$ photon energy range. The strongest spectral feature at a binding energy of $2.6 \mathrm{eV}$, for the highest $\mathrm{FOM} \mathrm{Cu} \mathrm{CrO}_{y}$ sample, is resonant between the photon energy of $48-50 \mathrm{eV}$. The intensity of this $\mathrm{Cr}$ $3 p-3 d$ resonance is broader and less intense by a factor of nearly two compared to the $\mathrm{Mg}_{x} \mathrm{Cr}_{2-x} \mathrm{O}_{3}$ film. The onset of resonance is composed of one broad peak in contrast to the $\mathrm{Mg}_{x} \mathrm{Cr}_{2-x} \mathrm{O}_{3}$ films, which has two distinct onsets of resonance. The disordered nature of the films and lack of long-range order would lead to a breakdown of crystal-field symmetry rules for an energetic splitting of the $\mathrm{t}_{2 g}$ and $\mathrm{e}_{g}$ levels, which leads to the absence of these features in all $\mathrm{Cu}_{x} \mathrm{CrO}_{y}$ films. Other than that detail the differential spectra shows striking similarity to the one of the crystalline $\mathrm{Mg}_{x} \mathrm{Cr}_{2-x} \mathrm{O}_{3}$ film, suggesting the importance of the chromium octahedra in the electronic composition of the valence band of the higher $\mathrm{FOM} \mathrm{Cu}_{x} \mathrm{CrO}_{y}$ sample.

These observations show that a strong chromium composition must occupy the top of the valence band for these three $p$-type films. This correlates well with the previous works by Yokobori et al. on crystalline $\mathrm{CuCrO}_{2}: \mathrm{Mg}$ films where the 

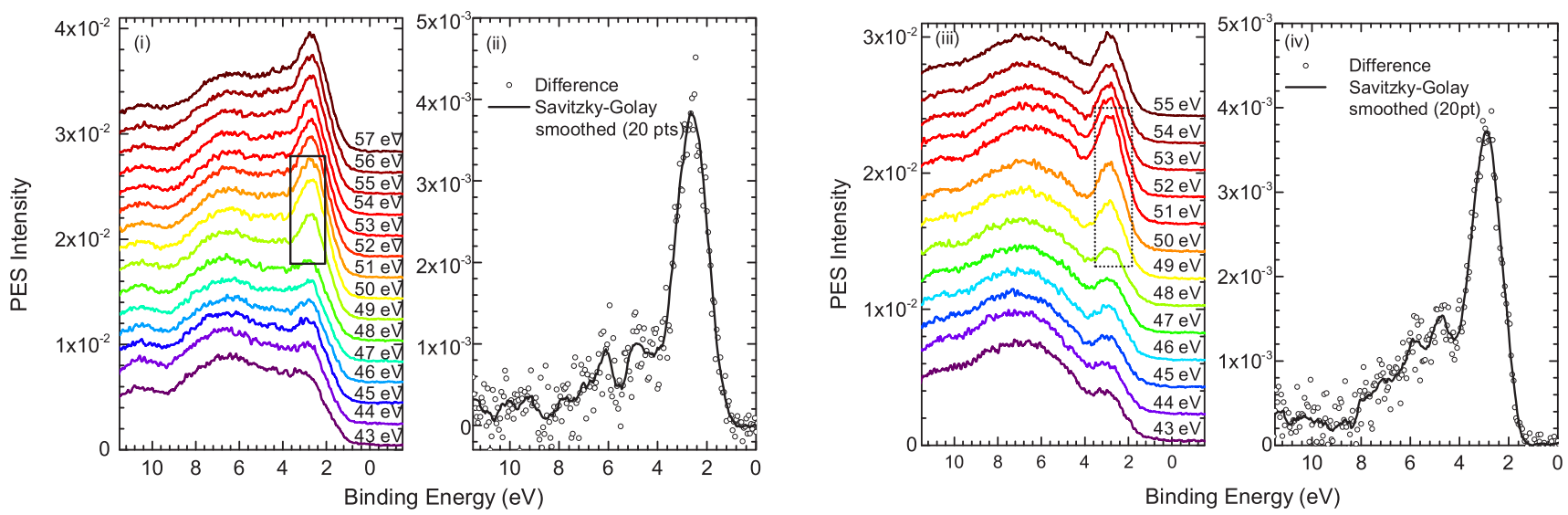

FIG. 6. The black box in (i) and (iii) indicates the region where resonant behavior is exhibited between 48 and $50 \mathrm{eV}$ in (i) and 48 and $51 \mathrm{eV}$ for (iii). Plots (ii) and (iv) show the difference spectra between $50-48 \mathrm{eV}$ in (ii) and $51-48 \mathrm{eV}$ in (iv). (ii) Peak fitting shows the sharp onset of resonance to be at a binding energy of $2.6 \mathrm{eV}$ with a FWHM $=1.6 \mathrm{eV}$ and an increase of PES intensity is 0.004 from $48-51$ eV at $2.6 \mathrm{eV}$ binding energy for the high $\mathrm{FOM} \mathrm{Cu}_{x} \mathrm{CrO}_{y}$ sample. (iv) The lower FOM sample shows the sharp onset of resonance to be at a binding energy of $3 \mathrm{eV}$ with a FWHM of $1.7 \mathrm{eV}$, with a PES intensity increase equal to 0.004 .

top of the valence band exhibits a strong chromium resonance around the $\mathrm{Cr} 3 p-3 d$ transition. For in situ cleaved samples, the increase in photoemission intensity at resonance was 0.003 , in line with the observed intensity changes in the copper deficient $\mathrm{Cu}_{x} \mathrm{CrO}_{y}$ films investigated here [28].

The $\mathrm{Cu} 3 d$ states are also expected in the valence-band region of $\mathrm{Cu}_{x} \mathrm{CrO}_{y}$ films. However, no copper resonance is seen, as outlined in Fig. 7. Stoichiometric, crystalline $\mathrm{CuCrO}_{2}: \mathrm{Mg}$ shows a weak resonance around the $\mathrm{Cu} 3 p-3 d$ transition, approximately a factor of four smaller than for the Cr $3 p$-3d transition [28]. In contrast to these measurements, the $\mathrm{Cu}_{x} \mathrm{CrO}_{y}$ films are substantially copper deficient and crystallographically disordered [34]. We also do not observe the two charge transfer satellites at 13 and $15 \mathrm{eV}$ corresponding to $\mathrm{Cu}^{+}\left(3 d^{10}\right)$ and $\mathrm{Cu}^{2+}\left(3 d^{9}\right)$ like initial states that Yokobori et al. observed. It would be expected that a broadening and reduced intensity, due to a lower copper content, could render the copper resonance and charge transfer satellites unresolvable in this case, while also being further complicated by the delocalized nature of copper $d$ states due to the on-site $3 d 4 s$ hybridization which introduces $4 s$ character into the valence band. Within the signal to noise ratio, the lack of any observed copper resonance sets an upper limit on any resonant behavior. This upper limit of a weak copper resonance and a strong chromium resonance lends some support to the proposed mechanism by Yokobori et al. that the ground state of $\mathrm{CuCrO}_{2}$ is showing signs of a $\mathrm{Cu} 4 s-\mathrm{Cr} 3 d$ charge transfer in $\mathrm{CuCrO}_{2}$ films via $\mathrm{O} 2 p$.

\section{Valence-band composition}

In order to assess the contribution of copper states qualitatively in the valence band of the $\mathrm{Cu}_{x} \mathrm{CrO}_{y}$ films, we can compare the shape of the valence-band PES spectra taken off-resonance at an incident photon energy of $85 \mathrm{eV}$ for samples with different copper content. The valence-band spectra are shown in Fig. 8, taken at an off-resonant photon energy of $85 \mathrm{eV}$ for both nanocrystalline $\mathrm{Cu}_{x} \mathrm{CrO}_{y}$ and crystalline $\mathrm{Mg}_{x} \mathrm{Cr}_{2-x} \mathrm{O}_{3}$ films. They have been compared with previous measurements on crystalline $\mathrm{CuCrO}_{2}: \mathrm{Mg}$ [28]. For a meaningful comparison, all spectra were aligned to the same valence-band maximum position of $\mathrm{CuCrO}_{2}: \mathrm{Mg}$; which coincided with the Fermi level at $0 \mathrm{eV}$ due to the high doping levels of the material. The absolute valence-band maximum
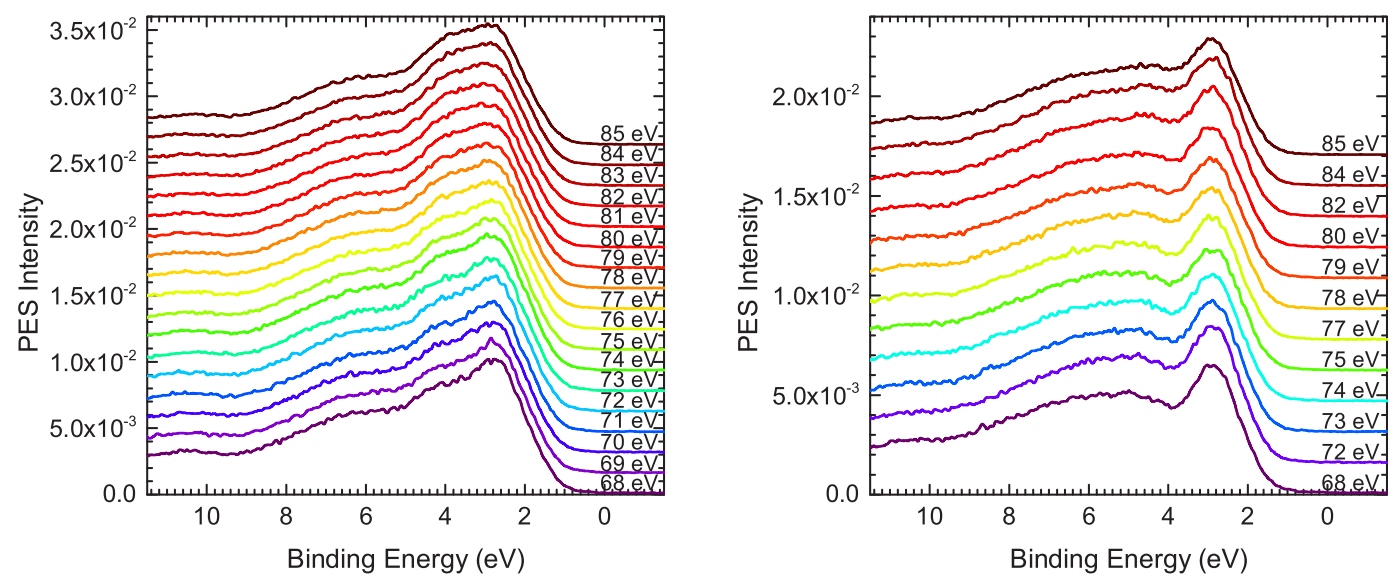

FIG. 7. Valence-band spectra of $\mathrm{Cu}_{x} \mathrm{CrO}_{y}$ films over the photon energies $68-85 \mathrm{eV}$, probing the copper $3 p-3 d$ resonance. 


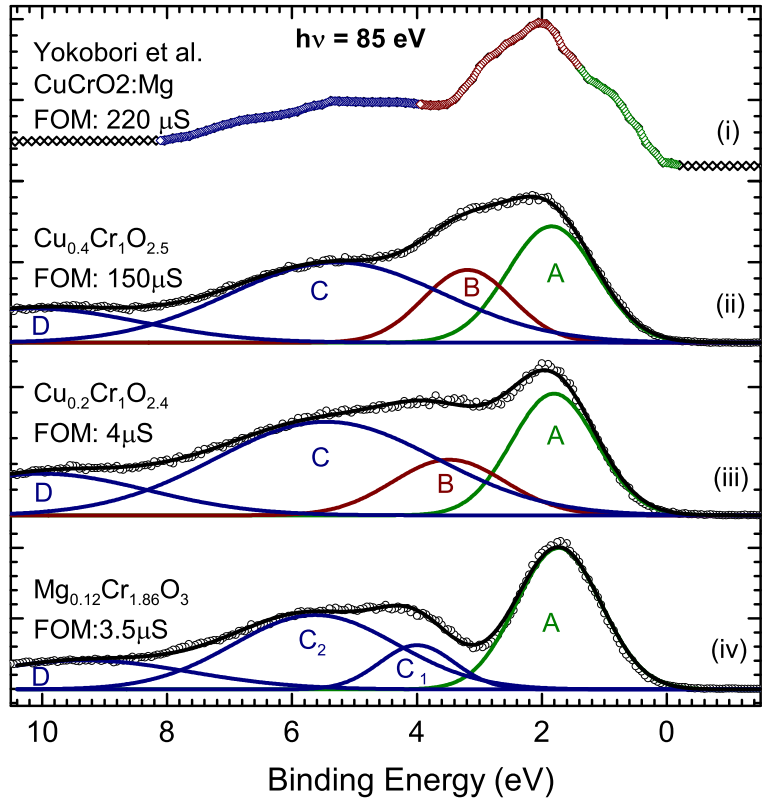

FIG. 8. Valence-band spectra of all p-type films compared at the photon energy of $85 \mathrm{eV}$. For comparison, all spectra have been aligned to the valence-band maximum of $\mathrm{CuCrO}_{2}: \mathrm{Mg}$ [28] to account for shifts in the Fermi level of the different oxide films.

position for $\mathrm{Cu}_{0.4} \mathrm{Cr}_{1} \mathrm{O}_{2.5}, \mathrm{Cu}_{0.4} \mathrm{Cr}_{1} \mathrm{O}_{2.5}$, and $\mathrm{Mg}_{0.12} \mathrm{Cr}_{1.86} \mathrm{O}_{3}$ lie $0.8,1$, and $1 \mathrm{eV}$ below the Fermi level (seen in Figs. 4 and 6).

Density functional theory (DFT) can model the composition of the valence-band spectra. In fact, studies have been carried out previously for $\mathrm{Cr}_{2} \mathrm{O}_{3}$ [46], $\mathrm{CuCrO}_{2}$ [26], and $\mathrm{CuCrO}_{2}: \mathrm{Mg}$ [28]. No in-depth study has been carried out for $p$-type $\mathrm{Mg}_{x} \mathrm{Cr}_{2-x} \mathrm{O}_{3}$. However, as mentioned previously, some discrepancy can arise about the best parameters to model a particular material. Experimental reports become crucial to providing qualitative evidence for modeling material properties.

Our measurements have been fitted with Gaussian peaks, in order to deconvolute prominent features in the VBM PES data. Best fits were obtained for all spectra containing four components. The dominant structure (peak A) in these fits is attributed to the $\mathrm{Cr} 3 d$ states, as the position and width coincides with the $\mathrm{Cr} 3 d$ partial density of states extracted from the differential valence-band PES (Figs. 4 and 6). This matches previous reports for both $\mathrm{CuCrO}_{2}: \mathrm{Mg}$ and $\mathrm{Cr}_{2} \mathrm{O}_{3}$ films $[28,46]$.

The spectral features of Yokobori et al. [spectrum (i) in Fig. 8], were assigned by DFT calculations in their report with predominant oxygen $p$ states lying between 4 and $8 \mathrm{eV}$ (blue). We assume the $\mathrm{O} 2 p$ states to lie in a similar energetic region for our $\mathrm{Cu}_{x} \mathrm{CrO}_{y}$ films. While all $\mathrm{Cu}_{x} \mathrm{CrO}_{y}$ films show a broad feature (labelled $\mathrm{C}$ ) in this energy range, the crystalline $\mathrm{Cr}_{2} \mathrm{O}_{3}$ sample does show a fine structure (labelled $\mathrm{C}_{1}$ and $\mathrm{C}_{2}$ ) consistent with the valence-band structure of undoped $\mathrm{Cr}_{2} \mathrm{O}_{3}$ [48]. A similar fine structure in the $\mathrm{Cr} 3 d$ states has not been resolved as a consequence of spectral broadening by the $\mathrm{Mg}$ incorporation.

As mentioned, across the copper $3 p-3 d$ threshold and upwards, no resonance was apparent in the studied $\mathrm{Cu}_{x} \mathrm{CrO}_{y}$ films. However, comparing the spectra shape of the different valence bands, an additional structure is apparent for the $\mathrm{Cu}$ containing, polycrystalline films (Fig. 8, labeled B), when compared with the $\mathrm{Mg}_{x} \mathrm{Cr}_{2-x} \mathrm{O}_{3}$ films. Energetically, the structure is found $1-2 \mathrm{eV}$ above the $\mathrm{Cr} 3 d$ states and is found to be more intense in the sample of higher copper content. This lies in a similar region to the calculations for the $\mathrm{Cu} 3 d$ states for crystalline $\mathrm{CuCrO}_{2}: \mathrm{Mg}$. If compared to the previously investigated crystalline material, all valence-band structures are significantly broadened, which is a consequence of the defective, nanocrystalline nature of the films. Equally, the ratio between the $\mathrm{Cr} 3 d$ and $\mathrm{Cu} 3 d$ intensity significantly differs from previous reports, as all films investigated here are copper deficient. In addition, the polycrystalline nature of thin films of glass will not have a coherent surface termination as in situ cleaved films, leading to the loss of fine structure in our measurements. It has been previously reported that the surface termination can significantly alter the valence-band shape in $\mathrm{CuCrO}_{2}[12]$.

\section{CONCLUSION}

Resonant photoelectron spectroscopy was performed on several high figure of merit $p$-type TCOs; $\mathrm{Mg}_{x} \mathrm{Cr}_{2-x} \mathrm{O}_{3}$ (3.5 $\mu \mathrm{S})$ and $\mathrm{Cu}_{x} \mathrm{CrO}_{y}(150 \mu \mathrm{S})$ thin films. The top of the valence band seems to be composed largely of $\mathrm{Cr} 3 d$ states in all the films and is not altered significantly with increasing copper content for $\mathrm{Cu}_{x} \mathrm{CrO}_{y}$ films. This provides experimental evidence for the position of elemental states in the valence band of oxides featuring octahedral coordinated $\mathrm{Cr}$ sites. The observations explain why many recently discovered $p$-type TCOs such as $\mathrm{Cr}_{2} \mathrm{O}_{3}: \mathrm{Mg}, \mathrm{LaCrO}_{3}: \mathrm{Sr}, \mathrm{Cr}_{2} \mathrm{MnO}_{4}: \mathrm{Li}$, and even highly defective $\mathrm{Cu}_{x} \mathrm{CrO}_{y}$ are showing similar good performance for $p$-type TCOs. The similarity of the $\mathrm{Cr} 3 d$ partial density of states for all the investigated films also highlights the limitation of creating dispersion in the valence band by cation doping, which leaves the $\mathrm{Cr}-\mathrm{O}_{6}$ octahedra unchanged.

Our findings highlight that for Cr-based $p$-type TCOs the valence-band structure is dominated by the presence of the $\mathrm{Cr}^{-} \mathrm{O}_{6}$ octahedra, independent of their long-range crystallographic order. This suggests that the $\mathrm{Cr} 3 d$ states are highly localized within the $\mathrm{Cr}-\mathrm{O}_{6}$ octahedra further confirming hopping mechanisms for carrier transport previously discussed for these materials $[21,31,49]$. To increase the hole mobility, it is likely that this $\mathrm{Cr}-\mathrm{O}_{6}$ octahedral coordination has to be altered, e.g., by anion doping or strain.

However, this particular valence-band composition allows for good performing, noncrystalline Cr-based $p$-TCOs, which can therefore be synthesized via low-temperature processes like spray pyrolysis. The example of nanocrystalline $\mathrm{Cu}_{x} \mathrm{CrO}_{y}$ illustrates that poor crystallinity is not necessarily detrimental to the figure of merit and opens new possibilities for applications were high hole mobilities are of less importance.

\section{ACKNOWLEDGMENTS}

This work was supported by the Irish Research Council under Grant No. GOI/PG/2013/445 and Science Foundation Ireland under Grant No. 12/IA/1264. Funding from the Higher 
Education Authority under the Programme for Research in Third-Level Institutions scheme, Cycle 5 is also gratefully acknowledged. The research leading to these results has received funding from the European Community's Seventh Frame- work Programme (FP7/2007-2013) under Grant agreement No. 312284. We express our thanks to the MAX laboratory beamline staff in particular, A. Preobrajenski, K. Handrup and A. Generalov, for their help and technical assistance.
[1] G.-H. Lee, Y.-J. Yu, X. Cui, N. Petrone, C.-H. Lee, M. S. Choi, D.-Y. Lee, C. Lee, W. J. Yoo, K. Watanabe et al., ACS Nano 7, 7931 (2013).

[2] S. De, T. M. Higgins, P. E. Lyons, E. M. Doherty, P. N. Nirmalraj, W. J. Blau, J. J. Boland, and J. N. Coleman, ACS Nano 3, 1767 (2009).

[3] H. Hosono, Thin Solid Films 515, 6000 (2007).

[4] A. Banerjee and K. Chattopadhyay, Prog. Cryst. Growth Charact. Mater. 50, 52 (2005).

[5] S. Sheng, G. Fang, C. Li, S. Xu, and X. Zhao, Physica Status Solidi (a) 203, 1891 (2006).

[6] J. Robertson, R. Gillen, and S. Clark, Thin Solid Films 520, 3714 (2012).

[7] G. Hautier, A. Miglio, G. Ceder, G.-M. Rignanese, and X. Gonze, Nat. Commun. 4, 2292 (2013).

[8] H. Yanagi, H. Tomomi, S. Ibuki, K. Ueda, and H. Hosono, Appl. Phys. Lett. 78, 1583 (2001).

[9] G. Thomas, Nature (London) 389, 907 (1997).

[10] S. Lany, J. Phys.: Condens. Matter 27, 283203 (2015).

[11] H. Kawazoe, M. Yasukawa, H. Hyodo, M. Kurita, H. Yanagi, and H. Hosono, Nature (London) 389, 939 (1997).

[12] D. Shin, J. S. Foord, R. G. Egdell, and A. Walsh, J. Appl. Phys. 112, 113718 (2012).

[13] M. O'Sullivan, P. Stamenov, J. Alaria, M. Venkatesan, and J. M. D. Coey, J. Phys.: Conf. Ser. 200, 052021 (2010).

[14] X. Xu, J. Bullock, L. T. Schelhas, E. Z. Stutz, J. J. Fonseca, M. Hettick, V. L. Pool, K. F. Tai, M. F. Toney, X. Fang et al., Nano Lett. (2016).

[15] R. Po, C. Carbonera, A. Bernardi, and N. Camaioni, Energy Environ. Sci. 4, 285 (2011).

[16] P. Qin, G. Fang, Q. He, N. Sun, X. Fan, Q. Zheng, F. Chen, J. Wan, and X. Zhao, Sol. Energy Mater. Sol. Cells 95, 1005 (2011).

[17] P. Qin, G. Fang, N. Sun, X. Fan, Q. Zheng, F. Chen, J. Wan, and X. Zhao, Thin Solid Films 519, 4334 (2011).

[18] L. Chen, J. Yang, S. Klaus, L. J. Lee, R. Woods-Robinson, J. Ma, Y. Lum, J. K. Cooper, F. M. Toma, L.-W. Wang et al., J. Am. Chem. Soc. 137, 9595 (2015).

[19] R. G. Gordon, MRS Bull. 25, 52 (2000).

[20] R. Nagarajan, N. Duan, M. Jayaraj, J. Li, K. Vanaja, A. Yokochi, A. Draeseke, J. Tate, and A. Sleight, Int. J. Inorgan. Mater. 3, 265 (2001).

[21] L. Farrell, K. Fleischer, D. Caffrey, D. Mullarkey, E. Norton, and I. V. Shvets, Phys. Rev. B 91, 125202 (2015).

[22] L. Farrell, E. Norton, B. J. O'Dowd, D. Caffrey, I. V. Shvets, and K. Fleischer, Appl. Phys. Lett. 107, 031901 (2015).

[23] S. Lim, S. Desu, and A. Rastogi, J. Phys. Chem. Solids 69, 2047 (2008).

[24] D. O. Scanlon, A. Walsh, B. J. Morgan, G. W. Watson, D. J. Payne, and R. G. Egdell, Phys. Rev. B 79, 035101 (2009).

[25] H. Hiraga, T. Makino, T. Fukumura, H. Weng, and M. Kawasaki, Phys. Rev. B 84, 041411 (2011).
[26] T. Arnold, D. J. Payne, A. Bourlange, J. P. Hu, R. G. Egdell, L. F. J. Piper, L. Colakerol, A. De Masi, P.-A. Glans, T. Learmonth et al., Phys. Rev. B 79, 075102 (2009).

[27] D. O. Scanlon and G. W. Watson, J. Mater. Chem. 21, 3655 (2011).

[28] T. Yokobori, M. Okawa, K. Konishi, R. Takei, K. Katayama, S. Oozono, T. Shinmura, T. Okuda, H. Wadati, E. Sakai et al., Phys. Rev. B 87, 195124 (2013).

[29] E. Arca, K. Fleischer, and I. V. Shvets, Appl. Phys. Lett. 99, 111910 (2011).

[30] N. Uekawa and K. Kaneko, J. Phys. Chem. 100, 4193 (1996).

[31] K. H. L. Zhang, Y. Du, A. Papadogianni, O. Bierwagen, S. Sallis, L. F. J. Piper, M. E. Bowden, V. Shutthanandan, P. V. Sushko, and S. A. Chambers, Adv. Mater. 27, 5191 (2015).

[32] H. Peng, A. Zakutayev, S. Lany, T. R. Paudel, M. d'Avezac, P. F. Ndione, J. D. Perkins, D. S. Ginley, A. R. Nagaraja, N. H. Perry et al., Adv. Func. Mater. 23, 5267 (2013).

[33] A. R. Nagaraja, K. H. Stone, M. F. Toney, H. Peng, S. Lany, and T. O. Mason, Chem. Mater. 26, 4598 (2014).

[34] L. Farrell, E. Norton, C. M. Smith, D. Caffrey, I. V. Shvets, and K. Fleischer, J. Mater. Chem. C 4, 126 (2016).

[35] K. Fleischer, D. Caffrey, L. Farrell, E. Norton, D. Mullarkey, E. Arca, and I. V. Shvets, Thin Solid Films 594, 245 (2015).

[36] E. Stavitski and F. M. de Groot, Micron 41, 687 (2010).

[37] M. Grioni, J. F. van Acker, M. T. Czyzyk, and J. C. Fuggle, Phys. Rev. B 45, 3309 (1992).

[38] S. L. Hulbert, B. A. Bunker, F. C. Brown, and P. Pianetta, Phys. Rev. B 30, 2120 (1984).

[39] J. P. Hu, D. J. Payne, R. G. Egdell, P.-A. Glans, T. Learmonth, K. E. Smith, J. Guo, and N. M. Harrison, Phys. Rev. B 77, 155115 (2008).

[40] M. Nolan and S. D. Elliott, Chem. Mater. 20, 5522 (2008).

[41] M. Brik, N. Avram, and C. Avram, Solid State Commun. 132, 831 (2004).

[42] P. S. Bagus, E. S. Ilton, and J. R. Rustad, Phys. Rev. B 69, 205112 (2004).

[43] H. Ikeno, T. Mizoguchi, and I. Tanaka, Phys. Rev. B 83, 155107 (2011).

[44] M. Matsubara, T. Uozumi, A. Kotani, and J. Claude Parlebas, J. Phys. Soc. Jpn. 74, 2052 (2005).

[45] J. Campbell and T. Papp, At. Data Nucl. Data Tables 77, 1 (2001).

[46] X. Li, L. Liu, and V. E. Henrich, Solid State Commun. 84, 1103 (1992).

[47] E. Bertel, R. Stockbauer, R. L. Kurtz, D. E. Ramaker, and T. E. Madey, Phys. Rev. B 31, 5580 (1985).

[48] S. Chambers, J. Williams, M. Henderson, A. Joly, M. Varela, and S. Pennycook, Surface Sci. 587, L197 (2005).

[49] A. Barnabé, Y. Thimont, M. Lalanne, L. Presmanes, and P. Tailhades, J. Mater. Chem. C 3, 6012 (2015). 